\title{
Micronutrient deficiencies in inflammatory bowel disease: trivial or crucial?
}

\author{
Soon Man Yoon \\ Department of Internal Medicine, Chungbuk National University Hospital, Chungbuk National University College of Medicine, Cheongju, Korea
}

\section{Article: Wernicke's encephalopathy after total parenteral nutrition in patients with Crohn's disease (Intest Res 2016;14:191-196)}

Patients with IBD, especially those with CD, are at a risk for a variety of nutritional deficiencies because of reduced nutrient intake or absorption, or increased nutrient losses. ${ }^{1}$ Nutritional deficiencies in IBD patients can be divided into those involving macronutrients (nutrients needed in large amounts) and those involving micronutrients (nutrients required in small quantities). ${ }^{2}$ In adults, deficiencies of micronutrients, including several water- and fat-soluble vitamins, and minerals including calcium, iron, and other trace minerals, are a common problem. ${ }^{3}$

Although the nutritional issues in IBD have become less common with advances in treatment and the greater proportions of patients attaining clinical remission, micronutrient deficiencies are still relatively common, particularly in CD patients with active small bowel disease or multiple resections. The major micronutrient deficiencies in IBD patients are associated with anemia (iron, folate, and vitamin B12), bone disease (calcium, vitamin $\mathrm{D}$, and possibly vitamin $\mathrm{K}$ ), hypercoagulability (folate, vitamin B6, and vitamin B12), wound healing (zinc, vitamin A, and vitamin C), and colorectal cancer risk (folate and possibly vitamin $\mathrm{D}$ and calcium). ${ }^{3}$ There are multiple mechanisms that can contribute to micronutrient deficiencies in IBD, such as decreased nutrient

Received March 11, 2016. Revised March 21, 2016.

Accepted March 23, 2016.

Correspondence to Soon Man Yoon, Department of Internal Medicine,

Chungbuk National University Hospital, Chungbuk National University

College of Medicine, 776, 1 Sunhwan-ro, Seowon-gu, Cheongju 28644,

Korea. Tel: +82-43-269-7241, Fax: +82-43-273-3252, E-mail: smyoon@ chungbuk.ac.kr

Financial support: None. Conflict of interest: None. intake, increased intestinal loss, malabsorption, hypermetabolic state, drug interactions, and long-term total parenteral nutrition (TPN). ${ }^{4}$

Numerous vitamin and mineral deficiencies have been reported in IBD patients, with varying degrees of clinical significance. ${ }^{2}$ Vitamins and minerals are naturally occurring compounds that are required for diverse bodily functions, and must be obtained from the diet as they are not sufficiently synthesized by humans. Vitamins are organic compounds that can be classified as either water-soluble or fat-soluble. Water-soluble vitamins include thiamine (B1), riboflavin (B2), nicotinic acid/niacin (B3), pyridoxine (B6), cobalamin (B12), biotin, pantothenic acid, folic acid, and vitamin $\mathrm{C}$ (ascorbic acid). The fat-soluble vitamins (A, D, E, and K) are hydrophobic substances that are dissolved within fat droplets and must be broken down by lipases and combined with bile salts in the duodenum to form mixed micelles. ${ }^{3}$

In the current article, the authors reported two patients with CD who developed Wernicke's encephalopathy (WE) after prolonged TPN. ${ }^{5} \mathrm{WE}$ is a serious neurologic complication due to vitamin B1 (thiamine) deficiency. Thiamine deficiency also can cause peripheral neuropathy and cardiomyopathy. Thiamine is found in multiple dietary sources (eggs, meats, bread, and nuts), and its absorption mainly occurs in the jejunum through varying degrees of active and passive transport, depending on body stores and the luminal concentrations of the vitamin. ${ }^{3}$ A recent study showed that thiamine deficiency may be more common in CD patients than in controls. ${ }^{6}$ This study was performed on 54 CD patients whose disease was in clinical remission, and were compared with 25 healthy controls. In CD patients, dietary

๑ Copyright 2016. Korean Association for the Study of Intestinal Diseases. All rights reserved.

This is an Open Access article distributed under the terms of the Creative Commons Attribution Non-Commercial License (http://creativecommons.org/licenses/by-nc/4.0)

which permits unrestricted non-commercial use, distribution, and reproduction in any medium, provided the original work is properly cited. 
thiamine intake was significantly lower than that in controls and low serum vitamin B1 was found in around one-third of CD patients.

Both of the two cases of the present report had extensive small bowel involvement and severe malnutrition state, with a very low BMI. ${ }^{5}$ Although multiple factors contribute to micronutrient deficiencies in IBD, some nutrients are digested and absorbed within specific locations in the gastrointestinal tract, and the activity and location of a patient's intestinal disease determines the risk for specific micronutrient deficiencies. Normally, more than $95 \%$ of vitamins and minerals from food are absorbed in the proximal small bowel, usually by the mid-jejunum. ${ }^{3}$ Patients who had undergone small bowel resection are also at a risk for micronutrient deficiencies, the nature of which depends on the extent and location of the resected bowel. ${ }^{7}$

The two patients of the present report had a relatively short duration of fasting and peripheral TPN (3 weeks and 17 days, respectively). ${ }^{5}$ As the authors mentioned, despite the relatively short period of fasting, the active inflammation caused by active $\mathrm{CD}$, underlying malnourished state, infection, and surgery led to an increased thiamine requirement and to the development of WE. The use of long-term parenteral nutrition can lead to deficiencies in any micronutrient not added in sufficient quantities, and the reported deficiencies include those of vitamins, minerals, and trace elements such as $\mathrm{Zn}^{2+}, \mathrm{Cu}^{2+}$, selenium, and chromium. ${ }^{3}$ Fortunately, the symptoms in the two patients of the current report resolved without any neurologic sequelae after thiamine replacement. ${ }^{5}$ As the authors also mentioned, WE is diagnosed clinically and is a disease with no characteristic and pathognomonic abnormalities in diagnostic studies. Thus, the development of neurologic disability in IBD patients with malnutrition should raise the suspicion of thiamine deficiency.

Micronutrient deficiencies, especially those of vitamins and essential trace elements, in IBD patients are common and associated with a complicated course of disease. IBD patients usually do not present overt symptoms of vitamin or micronutrient deficiency. Therefore, a high suspicion for micronutrient deficiencies is advocated for the timely treatment and prevention of irreversible complications.

\section{REFERENCES}

1. Weisshof R, Chermesh I. Micronutrient deficiencies in inflammatory bowel disease. Curr Opin Clin Nutr Metab Care 2015;18:576-581.

2. Massironi S, Rossi RE, Cavalcoli FA, Della Valle S, Fraquelli M, Conte D. Nutritional deficiencies in inflammatory bowel disease: therapeutic approaches. Clin Nutr 2013;32:904-910.

3. Hwang C, Ross V, Mahadevan U. Micronutrient deficiencies in inflammatory bowel disease: from A to zinc. Inflamm Bowel Dis 2012;18:1961-1981.

4. Wedrychowicz A, Zajac A, Tomasik P. Advances in nutritional therapy in inflammatory bowel diseases: Review. World J Gastroenterol 2016;22:1045-1066.

5. Shin IS, Seok H, Eun YH, et al. Wernicke's encephalopathy after total parenteral nutrition in patients with Crohn's disease. Intes Res 2016;14:191-196.

6. Filippi J, Al-Jaouni R, Wiroth JB, Hébuterne X, Schneider SM. Nutritional deficiencies in patients with Crohn's disease in remission. Inflamm Bowel Dis 2006;12:185-191.

7. Van Gossum A, Cabre E, Hébuterne X, et al. ESPEN Guidelines on Parenteral Nutrition: gastroenterology. Clin Nutr 2009;28:415-427. 